\title{
Strongly interacting one-dimensional Bose-Einstein condensates in harmonic traps
}

\author{
B. Tanatar and K. Erkan \\ Department of Physics, Bilkent University, Bilkent, 06533 Ankara, Turkey
}

(Received 21 May 2000; published 9 October 2000)

\begin{abstract}
We study the interaction effects on the condensates by considering a model of one-dimensional bosons. The harmonic external potential allows for the formation of a condensate in these systems. Using a densityfunctional theory-type formalism we obtain an equation describing the condensate wave function in the limit of very strong interactions between the bosons. We then consider a model of strongly interacting two-component system of bosons and study its stability conditions. Similar to the weakly interacting case, the two-component system exhibits coexisting and segregated phases depending on system parameters.
\end{abstract}

PACS number(s): 03.75.Fi, 05.30.Jp, 32.80.Pj

\section{INTRODUCTION}

The observation of Bose-Einstein condensation (BEC) phenomena in trapped atomic gases [1] has led to a surge of research activity. The thermodynamic, ground-state static and dynamic properties of condensates are thoroughly reviewed [2]. As the number of atoms in the condensates increases the deviations from the weak coupling description or the effects beyond the mean-field description will be more and more important, and this fact has been recognized earlier on $[3,4]$ and emphasized recently $[5,6]$. Another interesting direction in the recent studies of the BEC is the interplay between the dimensionality and interaction effects. The prospects of creating an effectively one- or two-dimensional condensates appears to be very optimistic.

Our aim in this work is to study some properties of a strongly interacting one-dimensional (1D) boson condensates in harmonic trap potentials. There are several motivations for undertaking such a study. First, in highly anisotropic trap potentials as used in the present experiments, the cigar shaped condensates are formed which may be modeled by a $1 \mathrm{D}$ equation [7]. It is also suggested [8] that thin atom waveguides may be used to realize 1D gas of impenetrable bosons. Second, the role of strong interaction effects may be studied in a model system where an exact solution to the homogeneous problem is known. We use the local-density approximation to describe the interaction effects on the condensate and obtain a new equation valid in the strong interaction limit $[9,10]$. Bose condensates with strong interactions have attracted considerable attention, and our calculations are intended to describe some of the ground-state properties of these systems.

The 1D bosons have been the subject of various works in recent years [7-12]. Pearson, Pang, and Chen [13] studied the interacting Bose gas in 1D subject to power-law potentials employing the path-integral Monte Carlo method. They found that a macroscopically large number of particles occupy the lowest single-particle state in a finite system of hard-core bosons at some critical temperature. The validity of the Gross-Pitaevskii (GP) [14] equation even at zero temperature when the interactions are strong is an important question in our understanding of the boson condensates. The $1 \mathrm{D}$ condensates are usually modeled by a suitable averaging over the cross section and identifying the renormalized pa- rameters within the GP description [7]. In the strongly interacting limit, 1D bosons are described by a new type of nonlinear Schrödinger equation (with a fifth order nonlinearity) as discussed by Tanatar [9] and Kolomeisky et al. [10]. The strong interaction limit corresponds to the infinite hard-core problem envisaged by Girardeau [15]. The correspondence with noninteracting spinless fermions are noted and solitonic properties are investigated $[9,10]$. Recently, Girardeau and Wright [16], by studying the time evolution of the condensates when the external potential is turned off, found that coherence in strongly interacting bosons is lost. We also consider in this work the mixture of 1D Bose condensates and study their phase segregation properties. In our model calculations we investigate the effect of the external potential on the stability of the overlapping condensates in a twocomponent system.

The rest of this paper is organized as follows. In Sec. II, we obtain the nonlinear Schrödinger equation for 1D condensates in the strong coupling limit, and discuss the main features of the condensate wave function. In Sec. III, we study a model of a strongly interacting two-component system and study its stability conditions. We conclude in Sec. IV with a brief summary of our results.

\section{ONE-DIMENSIONAL BOSE CONDENSATES}

We consider a system of interacting bosons in $1 \mathrm{D}$ confined in a harmonic potential $V_{\text {ext }}(x)=\frac{1}{2} m \omega^{2} x^{2}$. To describe the dynamics of the condensate in both the weak and the strong coupling regimes we use the method developed by Nunes [6]. In this approach the energy functional for the condensate is written in the spirit of local-density approximation in terms of the ground-state energy of the homogeneous system. The nonlinear equation for the condensate wave function then follows as the Kohn-Sham equation. Starting from the energy functional

$$
E=\int_{-\infty}^{\infty} d x\left\{\frac{\hbar^{2}}{2 m}\left|\frac{d \psi}{d x}\right|^{2}+\frac{1}{2} m \omega^{2} x^{2}|\psi|^{2}+\epsilon(\rho)|\psi|^{2}\right\}
$$

where $\rho=|\psi|^{2}$ is the density and $\epsilon(\rho)$ is the ground-state energy (per particle) of the homogeneous system (i.e., in the 
absence of an external potential). Minimization of the total energy functional subject to the normalization condition yields

$$
\left\{-\frac{\hbar^{2}}{2 m} \frac{d^{2}}{d x^{2}}+\frac{1}{2} m \omega^{2} x^{2}+\frac{\partial(\epsilon \rho)}{\partial \rho}\right\} \psi=\mu \psi
$$

where $\mu$ is the chemical potential. The problem of $1 \mathrm{D}$ bosons interacting via a short range ( $\delta$-function) potential of arbitrary strength has been solved exactly by Lieb and Liniger [17]. In particular, the ground-state energy is given by $\epsilon(\rho)=g \rho / 2+\ldots$, where $g$ is the dimensionless coupling strength. The expression for $\epsilon(\rho)$ is also known as the Bogoliubov result valid at small $g$. Thus, Eq. (2) with weak coupling expression for the energy of the homogeneous system becomes the well-known Gross-Pitaevskii equation with a cubic nonlinear term. The properties of 1D condensates have been the subject of many works [7] in the weak coupling regime, where the coupling strength $g$ is treated as a parameter. The calculations show that a condensate cloud of a certain size depending on $g$ and $N$ exists at zero temperature because of the external potential.

We now turn to the limit of very strong coupling between the interacting bosons. For large coupling strengths the energy density is given by $\epsilon=\left(\pi^{2} / 6\right) g^{2} /(g / \rho+2)^{2}$, and in particular, when $g$ is infinite, it simply reduces to $\epsilon=\pi^{2} \rho^{2} / 6$. Using the above Kohn-Sham form of the equation of motion we obtain

$$
\left\{-\frac{\hbar^{2}}{2 m} \frac{d^{2}}{d x^{2}}+\frac{1}{2} m \omega^{2} x^{2}+\frac{\pi^{2}}{2}|\psi|^{4}\right\} \psi=\mu \psi,
$$

for the condensate wave function in the strongly coupled limit. Interestingly, the new nonlinear equation contains a fifth order term, and the cubic term is altogether missing. The above fifth order nonlinear Schrödinger equation was also obtained by Kolomeisky et al. [10]. In an earlier work Kolomeisky and Straley [18] argue from a renormalization group analysis that the correct local-density theory predicts Eq. (2) for 1D impenetrable bosons. It was noted [10] that because of the boson-fermion equivalence $[15,17]$ exact density profiles can be obtained and the accuracy of the present mean-field approach can be tested. The fifth order nonlinear Schrödinger equation is found to describe the condensate density quite well for large $N$. We also mention here that according to Girardeau's theorem [15] an infinitely strong repulsion between bosons in one-dimension effectively turns the particles into noninteracting spinless fermions, as demonstrated by Lieb and Liniger [17]. Girardeau's theorem is exactly satisfied (at the Thomas-Fermi approximation level) for infinitely strongly coupled 1D bosons in an external potential, which was shown in the previous works $[9,10]$.

In Fig. 1 we display the numerical solution of Eq. (3) for several values of $N$ for the condensate wave function in the strong coupling limit. We use the harmonic oscillator length $a_{\mathrm{HO}}=(\hbar / m \omega)^{1 / 2}$ for scaling, thus the condensate wave function is rendered dimensionless by $\psi(x) \rightarrow\left(N / a_{\mathrm{HO}}\right)^{1 / 2} \psi(x)$. Remarkably, a condensate seems to exist in the sense that a finite cloud extends over a finite length. The corresponding

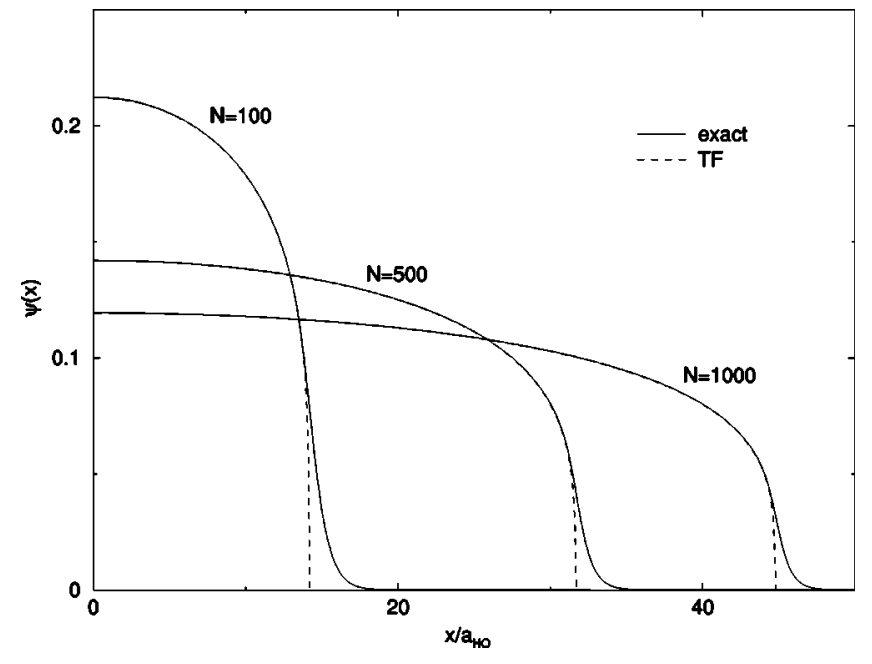

FIG. 1. Condensate wave function (in dimensionless units) in the infinite coupling limit for $N=100, N=500$, and $N=1000$ particles. The corresponding Thomas-Fermi results are indicated by dotted lines.

Thomas-Fermi (TF) approximation to Eq. (3), straightforwardly obtained by neglecting the kinetic energy term, is also plotted in Fig. 1, and we observe that it is quite accurate for a range of $x$ values except at the edge of the condensate. Using the argument [19] that the balance between the interaction energy $\left(\hbar^{2} / 2 m\right) \pi^{2}(N / R)^{2}$, and the confinement energy $m \omega^{2} R^{2}$, to determine the size of the condensate $R$, we estimate it to be $R / a_{\mathrm{HO}}=(\pi N)^{1 / 2}$ which is in good agreement with the numerical results shown in Fig. 1.

The strong coupling effects in condensed Bose systems have been addressed by a number of authors. Ziegler and Shukla [20], using the slave-boson technique, found that the effective potential grows only linearly in the asymptotic limit in contrast to the $\psi^{4}$ behavior in the GP functional. Thus, the condensate becomes weaker and is easily destroyed by fluctuation effects. Cherny and Shanenko [21] proposed a strong coupling generalization of the Bogoliubov model. Our results indicate that a condensate exists even for an infinitely strongly interacting $1 \mathrm{D}$ model system. It is interesting to note that recently Dodd et al., Eleftheriou and Huang, and Gammal et al. [22] have found a $\psi^{5}$ correction term to the GP equation by including three-body scattering effects in the 3D inhomogeneous systems. It would also be interesting to check our results for a strongly interacting bosons with a short-range potential in 1D (also under an external potential) by Monte Carlo calculations to see if the new type of nonlinear Schrödinger equation correctly describes the properties of the condensate. Recent calculations by Giorgini, Boronat, and Casulleras [23] could easily be extended to study the system considered here.

\section{TWO-COMPONENT SYSTEM}

Following the realization of Bose-Einstein condensation in single-component atomic vapors, experimental efforts succeeded in creating overlapping condensate mixtures [24]. The phase and density dynamics of two-component boson mixtures have been investigated in a gas of ${ }^{87} \mathrm{Rb}$ atoms with two hyperfine states [25]. Theoretical work concentrated on the study of the various properties of two-species Bose con- 

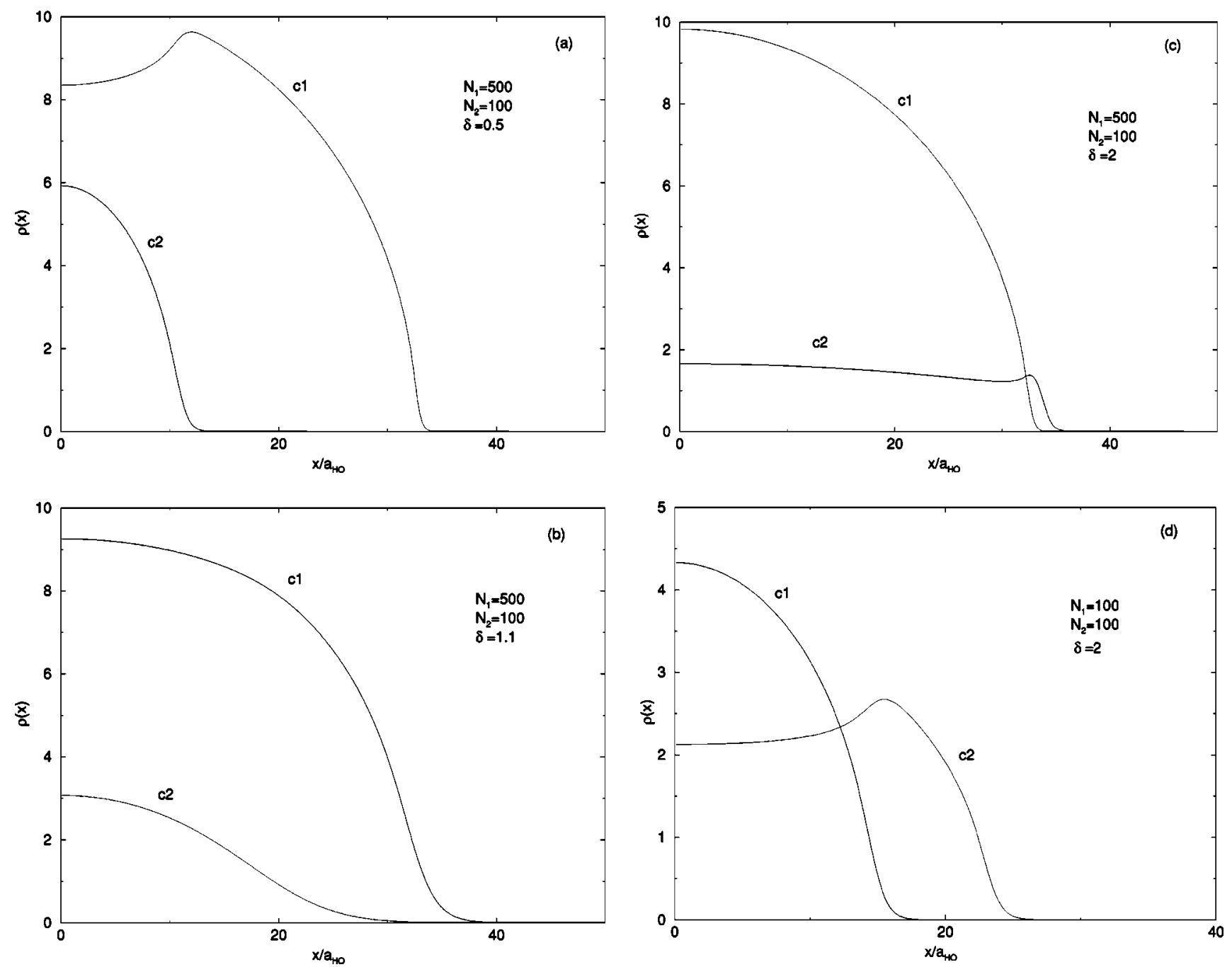

FIG. 2. Density profiles (in dimensionless units) of a two-component strongly interacting Bose system. Number of particles of each species and the mass ratio $\delta=m_{1} / m_{2}$ are indicated for each panel. The parameters are so chosen to show mostly overlapping condensates.

densates [26-30]. The stability of the boson mixtures against phase separation, the behavior of collective excitations, and tunneling effects between the condensates are investigated. In the case of 1D condensates Girardeau and Wright [16] considered interference effects when the confining potential is switched off, and found that coherence properties are largely lost.

To study some properties of strongly interacting boson mixtures, we consider a simple model of two species with different masses, i.e., $m_{1}$ and $m_{2}$, respectively. The total energy functional is expressed as

$$
\begin{aligned}
E\left[\psi_{1}, \psi_{2}\right]= & \int_{-\infty}^{\infty} d x\left\{\frac{\hbar^{2}}{2 m_{1}}\left|\frac{d \psi_{1}}{d x}\right|^{2}+\frac{\hbar^{2}}{2 m_{2}}\left|\frac{d \psi_{2}}{d x}\right|^{2}\right. \\
& +V_{1}^{\mathrm{ext}}\left|\psi_{1}\right|^{2}+V_{2}^{\mathrm{ext}}\left|\psi_{2}\right|^{2}+\epsilon\left(\rho_{1}\right) \rho_{1} \\
& \left.+\epsilon\left(\rho_{2}\right) \rho_{2}+\epsilon\left(\rho_{1}\right) \rho_{2}+\epsilon\left(\rho_{2}\right) \rho_{1}\right\}
\end{aligned}
$$

where $V_{i}^{\text {ext }}=\frac{1}{2} m_{i} \omega^{2} x^{2}$ are the external harmonic potentials (for simplicity we choose the same frequency), and $\rho_{i}$ $=\left|\psi_{i}\right|^{2}$ are the densities. In more realistic cases the external potential frequencies may be allowed to be different, and effects of gravity may be included. The coupled nonlinear equations that the Bose condensates satisfy are given as

$$
\begin{aligned}
& \left\{-\frac{\hbar^{2}}{2 m_{1}} \frac{d^{2}}{d x^{2}}+\frac{1}{2} m_{1} \omega^{2} x^{2}+\frac{\hbar^{2}}{2 m_{1}} \pi^{2}\left|\psi_{1}\right|^{4}\right. \\
& \left.+\frac{\hbar^{2}}{2 m_{1}} \frac{2 \pi^{2}}{3}\left|\psi_{1}\right|^{2}\left|\psi_{2}\right|^{2}+\frac{\hbar^{2}}{2 m_{2}} \frac{\pi^{2}}{3}\left|\psi_{2}\right|^{4}\right\} \psi_{1}=\mu_{1} \psi_{1}, \\
& \left\{-\frac{\hbar^{2}}{2 m_{2}} \frac{d^{2}}{d x^{2}}+\frac{1}{2} m_{2} \omega^{2} x^{2}+\frac{\hbar^{2}}{2 m_{2}} \pi^{2}\left|\psi_{2}\right|^{4}\right. \\
& \left.+\frac{\hbar^{2}}{2 m_{2}} \frac{2 \pi^{2}}{3}\left|\psi_{2}\right|^{2}\left|\psi_{1}\right|^{2}+\frac{\hbar^{2}}{2 m_{1}} \frac{\pi^{2}}{3}\left|\psi_{1}\right|^{4}\right\} \psi_{2}=\mu_{2} \psi_{2} .
\end{aligned}
$$

Note that the coupled nonlinear equations describing strongly interacting condensates are different in structure 

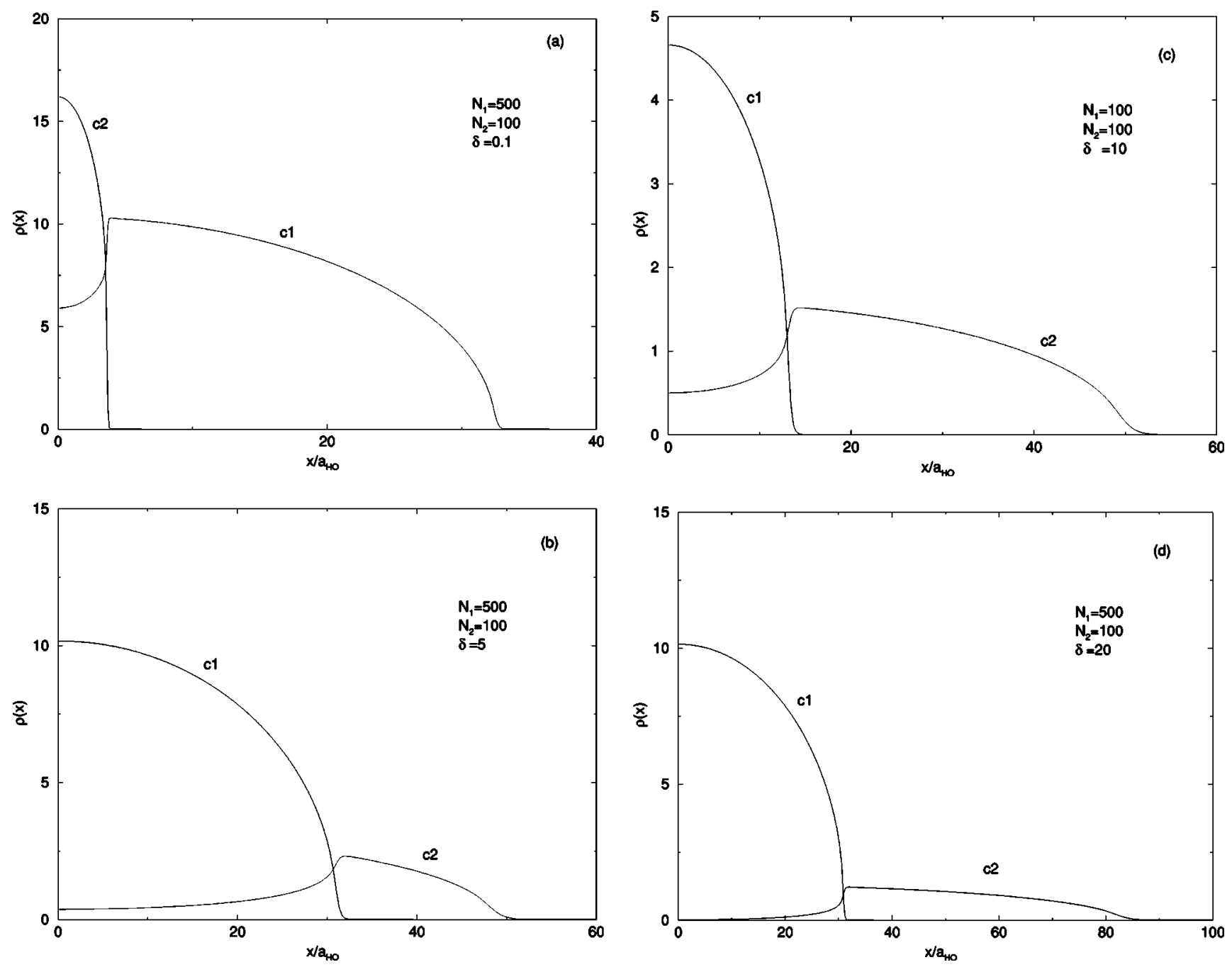

FIG. 3. Density profiles (in dimensionless units) of a two-component strongly interacting Bose system. Number of particles of each species and the mass ratio $\delta=m_{1} / m_{2}$ are indicated for each panel. The parameters are so chosen to show mostly segregated condensates.

than their counterparts in the weakly interacting regime. First, in contrast to the weak interaction limit we do not have interaction strengths appearing in the nonlinear equations. Second, various terms in the energy functional, for instance the interaction terms for the condensate of the same species and those for different species, contribute to yield terms with various powers of $\psi_{1}$ and $\psi_{2}$. This is also at variance with the weakly interacting system expressions, where there is only one coupling term in the coupled nonlinear equations. In the various panels of Figs. 2 and 3, we display the condensate density profiles of the two species for different combinations of $N_{1}, N_{2}$, and the mass ratio $\delta=m_{1} / m_{2}$. We have used the steepest descent method to solve numerically the coupled nonlinear equations for $\psi_{1}$ and $\psi_{2}$. Depending on the parameters chosen we find that the condensates either overlap in the same region of space, or show a segregated behavior. To better understand the stability of the homogeneous phase of the two-component system (i.e., overlapping condensates), we write down the total energy of the system as

$$
\begin{aligned}
E_{\mathrm{hom}}= & {\left[\frac{\hbar^{2}}{2 m_{1}} \frac{\pi^{2}}{3} \rho_{1}^{3}+\frac{\hbar^{2}}{2 m_{2}} \frac{\pi^{2}}{3} \rho_{2}^{3}\right.} \\
& \left.+\frac{\hbar^{2}}{2 m_{1}} \frac{\pi^{2}}{3} \rho_{1}^{2} \rho_{2}+\frac{\hbar^{2}}{2 m_{2}} \frac{\pi^{2}}{3} \rho_{1} \rho_{2}^{2}\right] L,
\end{aligned}
$$

where $\rho_{i}=\psi_{i}^{2}=N_{i} / L$. In the above expression, we have neglected the contribution of kinetic energy terms (TF approximation) and we have assumed that the trap potential is in the form of an infinite square well with size $L$. The homogeneous solution would be stable provided

$$
\frac{\partial^{2} E}{\partial \rho_{1}^{2}} \frac{\partial^{2} E}{\partial \rho_{2}^{2}}-\left(\frac{\partial^{2} E}{\partial \rho_{1} \partial \rho_{2}}\right)^{2}>0,
$$

which yields $1 / 3<\delta<3$. Outside this range of values for $\delta$, the condensates are in the phase separated state. The density profiles depicted in Figs. 2 and 3 illustrate these coexisting and segregated phases for various values of the mass ratio $\delta$. 
Similar qualitative stability bounds also follow by considering the total energy of the inhomogeneous state

$$
E_{\text {inhom }}=\frac{\hbar^{2}}{2 m_{1}} \frac{\pi^{2}}{3} \rho_{1}^{3} L_{1}+\frac{\hbar^{2}}{2 m_{2}} \frac{\pi^{2}}{3} \rho_{2}^{3} L_{2},
$$

where $L_{i}$ is the spatial extent of each condensate. Minimizing $E_{\text {inhom }}$ with respect to $L_{1}$ and $L_{2}$, under the constraint $L_{1}$ $+L_{2}=L$, we obtain

$$
\begin{gathered}
L_{1} / L=\left[1+\delta^{1 / 3} N_{2} / N_{1}\right]^{-1}, \\
L_{2} / L=\left[1+\delta^{-1 / 3} N_{1} / N_{2}\right]^{-1} .
\end{gathered}
$$

Although the numerical results presented in Figs. 2 and 3 are for harmonic confining wells, our approximate mean-field treatment results are consistent with the observed behavior. Our results are qualitatively similar to those found in 3D condensate mixtures, but the shape of the density profiles are determined by the strong coupling equations. Another important difference is that in 3D condensates the relative strength of the interparticle interactions among the species are responsible for the occurrence of different phases, whereas in the present case only the mass ratio is capable of producing similar results. The model of a strongly interacting twocomponent Bose condensate, thus, provides a useful example to study the different phases of condensate mixtures.

\section{SUMMARY}

In this work we have considered the ground-state properties of strongly interacting $1 \mathrm{D}$ boson condensates at zero temperature. Although it is a matter of debate that, the interaction effects do not destroy the condensate immediately according to the Monte Carlo simulations performed at finite temperature of Pearson, Pang, and Chen [13]. A finite temperature analysis (finding a nonzero critical temperature $T_{c}$ ) is necessary to establish the occurrence of a true BEC. Our study was at zero temperature; thus, it does not address the transition to a Bose-Einstein condensed phase. The solutions of the 1D-GP equation, however, indicate the formation of spatial distribution of bosons of a finite size which may be construed as a condensate cloud. Using the exact solution of the 1D homogeneous system of interacting bosons, we construct a new nonlinear equation to describe the condensate wave function in the strong coupling regime. The mixture of two such condensates in the strongly interacting regime yields stability conditions similar to the weakly interacting two-component $3 \mathrm{D}$ condensates. It would be interesting to extend our ideas to strongly interacting binary boson-fermion mixtures. From an experimental point of view, it would be useful to probe the strong interaction regime in the 1D or quasi-1D condensates.

\section{ACKNOWLEDGMENTS}

This work was partially supported by the Scientific and Technical Research Council of Turkey (TUBITAK) under Grant No. TBAG-1662, by NATO under Grant No. SfP971970, and by the Turkish Department of Defense under Grant No. KOBRA-001.
[1] M. H. Anderson, J. R. Ensher, M. R. Matthews, C. E. Wieman, and E. A. Cornell, Science 269, 198 (1995); K. B. Davis, M.-O. Mewes, M. R. Andrews, N. J. van Druten, D. S. Durfee, D. M. Kurn, and W. Ketterle, Phys. Rev. Lett. 75, 3969 (1995); C. C. Bradley, C. A. Sackett, and R. G. Hulet, Phys. Rev. Lett. 78, 985 (1997).

[2] F. Dalfovob S. Giorgini, L. Pitaevskii, and S. Stringari, Rev. Mod. Phys. 71, 463 (1999); A. S. Parkins and D. F. Walls, Phys. Rep. 303, 1 (1998).

[3] E. Timmermans, P. Tommasini, and K. Huang, Phys. Rev. A 55, 3645 (1997).

[4] E. Braaten and A. Nieto, Phys. Rev. B 56, 14745 (1997); 55, 8090 (1997).

[5] A. Fabrocini and A. Polls, Phys. Rev. A 60, 2319 (1999).

[6] G. S. Nunes, J. Phys. B 32, 4293 (1999).

[7] V. M. Pérez-García, H. Michinel, and H. Herrero, Phys. Rev. A 57, 3837 (1998); Y. S. Kivshar and T. J. Alexander, e-print cond-mat/9905048.

[8] M. Olshanii, Phys. Rev. Lett. 81, 938 (1998).

[9] B. Tanatar, Europhys. Lett. 51, 261 (2000).

[10] E. B. Kolomeisky, T. J. Newman, J. P. Straley, and X. Qi, Phys. Rev. Lett. 85, 1146 (2000).

[11] T. Haugset and H. Haugerud, Phys. Rev. A 57, 3809 (1998).

[12] H. Monien, M. Linn, and N. Elstner, Phys. Rev. A 58, 3395 (1998).
[13] S. Pearson, T. Pang, and C. Chen, Phys. Rev. A 58, 1485 (1998); see also, W. J. Mullin, J. Low Temp. Phys. 110, 167 (1998).

[14] E. P. Gross, Nuovo Cimento 20, 454 (1961); L. P. Pitaevskii, Zh. Éksp. Teor. Fiz. 40, 646 (1961) [JETP Lett. 13, 451 (1961)].

[15] M. Girardeau, J. Math. Phys. 1, 516 (1960).

[16] M. D. Girardeau and E. M. Wright, Phys. Rev. Lett. 84, 5239 (2000).

[17] E. H. Lieb and W. Liniger, Phys. Rev. 130, 1605 (1963).

[18] E. B. Kolomeisky and J. P. Straley, Phys. Rev. B 46, 11749 (1992).

[19] G. Baym and C. Pethick, Phys. Rev. Lett. 76, 6 (1996).

[20] K. Ziegler and A. Shukla, Phys. Rev. A 57, 1464 (1998).

[21] A. Yu. Cherny and A. A. Shanenko, e-print cond-mat/9904217.

[22] R. J. Dodd, M. Edwards, C. J. Williams, C. W. Clark, M. J. Holland, P. A. Ruprecht, and K. Burnett, Phys. Rev. A 54, 661 (1996); A. Eleftheriou and K. Huang, ibid. 61, 3601 (2000); A. Gammal, T. Frederico, L. Tomio, and Ph. Chomaz, e-print cond-mat/9911089.

[23] S. Giorgini, J. Boronat, and J. Casulleras, Phys. Rev. A 60, 5129 (1999).

[24] C. J. Myatt, E. A. Burt, R. W. Ghrist, E. A. Cornell, and C. E. 
Wieman, Phys. Rev. Lett. 78, 586 (1997).

[25] D. S. Hall, M. R. Matthews, J. R. Ensher, C. E. Wieman, and E. A. Cornell, Phys. Rev. Lett. 81, 1539 (1998); D. S. Hall, M. R. Matthews, C. E. Wieman, and E. A. Cornell, ibid. 81, 1543 (1998).

[26] T.-L. Ho and V. B. Shenoy, Phys. Rev. Lett. 77, 3276 (1996).

[27] H. Pu and N. P. Bigelow, Phys. Rev. Lett. 80, 1130 (1998); 80,
1134 (1998); C. K. Law, H. Pu, N. P. Bigelow, and J. H. Eberly, Phys. Rev. Lett. 79, 3105 (1997).

[28] E. P. Bashkin and A. V. Vagov, Phys. Rev. B 56, 6207 (1997); P. Öhberg, Phys. Rev. A 59, 634 (1999).

[29] P. Ao and S. T. Chui, Phys. Rev. A 58, 4836 (1998); J. Phys. B 33, 535 (2000).

[30] A. Sinatra and Y. Castin, Eur. Phys. J. D 8, 3129 (2000). 